\title{
Formation of Solid Electrolyte Interphase in Hydrate-Melt Electrolytes
}

\author{
Seongjae $\mathrm{Ko}^{1}$, Yuki Yamada ${ }^{1,2}$, and Atsuo Yamada, ${ }^{1,2}$ \\ 1. Department of Chemical System Engineering, The University of Tokyo, 7-3-1, Hongo, \\ Bunkyo-ku, Tokyo 113-8656, Japan \\ 2. Elements Strategy Initiative for Catalysts \& Batteries (ESICB), Kyoto University, 1-30, \\ Goryo-Ohara, Nishikyo-ku, Kyoto 615-8245, Japan
}

Corresponding Author*E-mail: yamada@chemsys.t.u-tokyo.ac.jp 

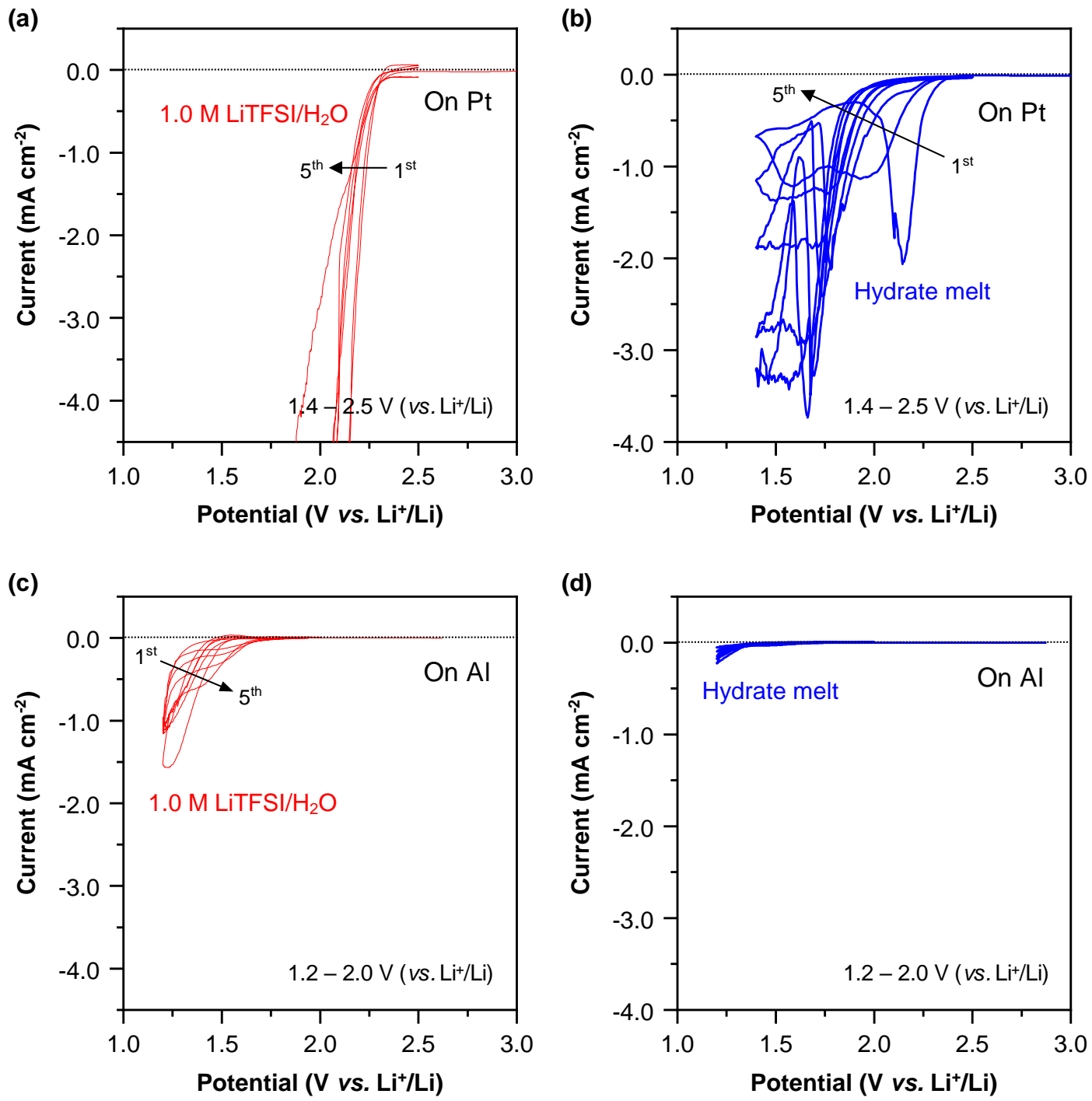

Figure S1. Reductive stability of dilute $1.0 \mathrm{M} \mathrm{LiTFSI} / \mathrm{H}_{2} \mathrm{O}$ and $\mathrm{Li}(\mathrm{TFSI})_{0.7}(\mathrm{BETI})_{0.3} \cdot 2 \mathrm{H}_{2} \mathrm{O}$ hydrate-melt electrolytes on the (a-b) Pt and (c-d) Al electrodes evaluated via cyclic voltammetry at scan rate of $0.1 \mathrm{mV} \mathrm{s}^{-1}$ for 5 cycles. 


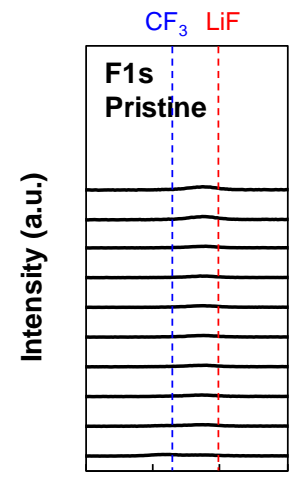

$695 \quad 690 \quad 685 \quad 680$

Binding energy (eV)

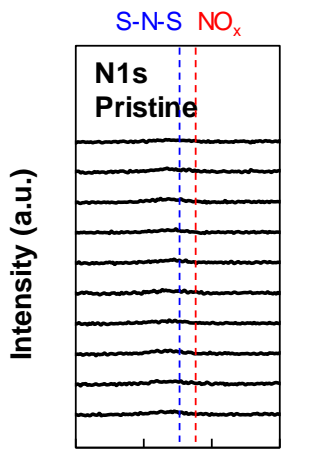

$407 \quad 402 \quad 397 \quad 392$

Binding energy (eV)

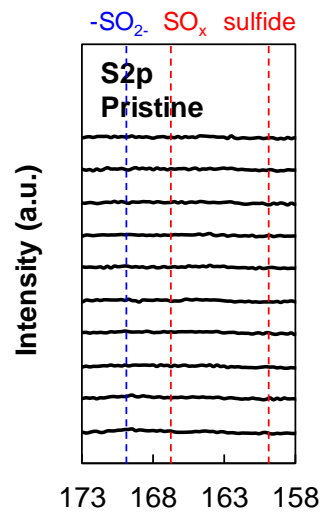

Binding energy (eV)

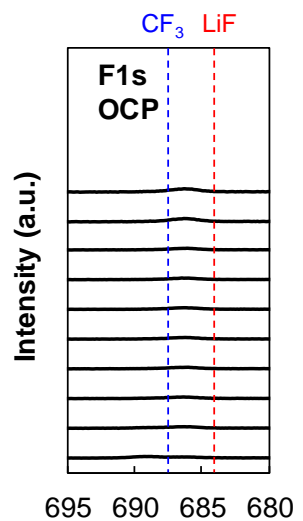

Binding energy (eV)

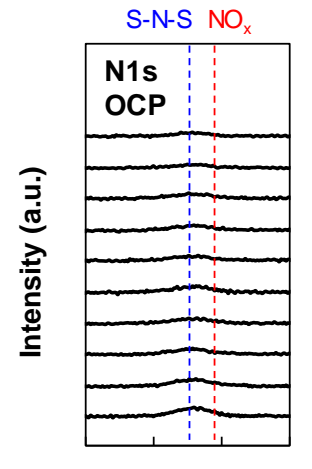

$407 \quad 402 \quad 397 \quad 392$

Binding energy (eV)

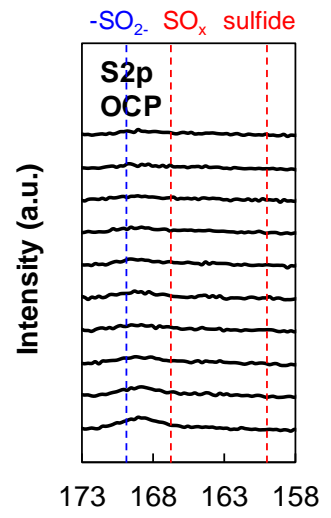

Binding energy (eV)

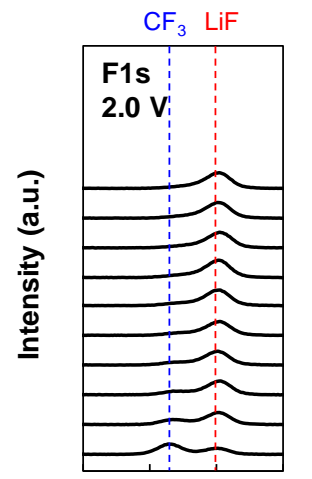

$695 \quad 690 \quad 685 \quad 680$

Binding energy (eV)

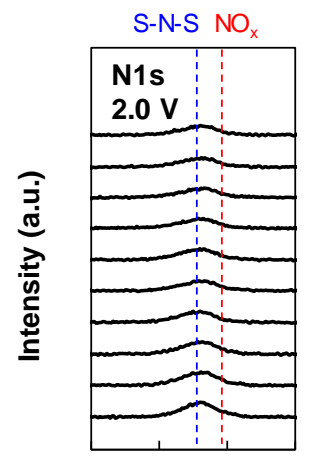

$407 \quad 402 \quad 397 \quad 392$

Binding energy (eV)

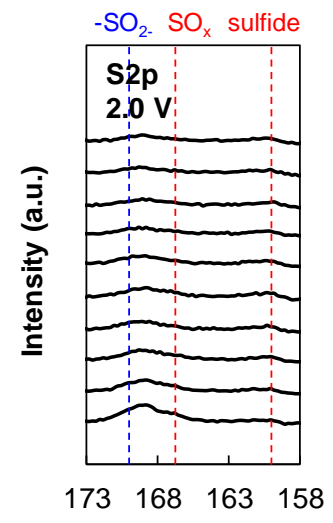

Binding energy (eV)

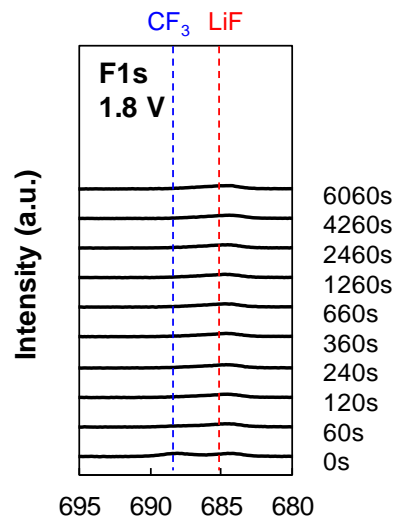

Binding energy (eV)

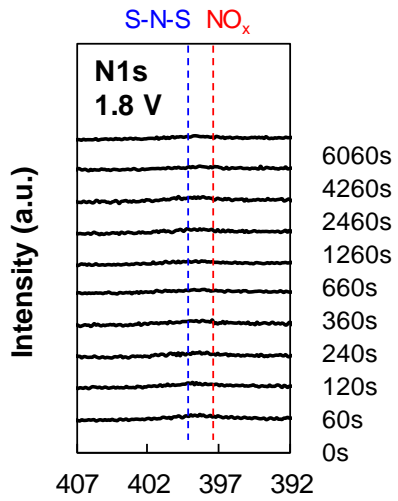

Binding energy (eV)

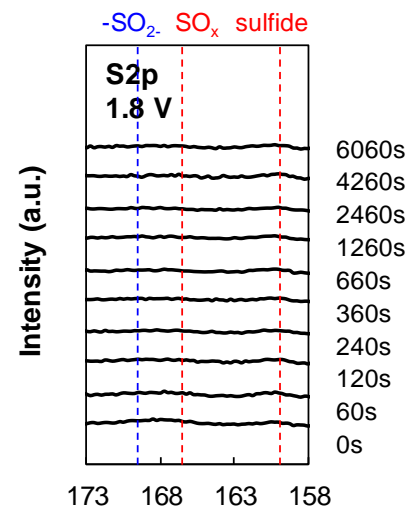

Binding energy (eV) 


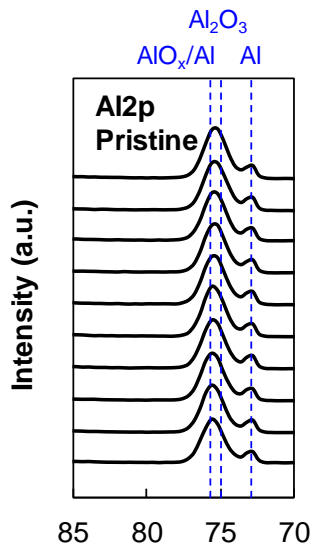

Binding energy (eV)

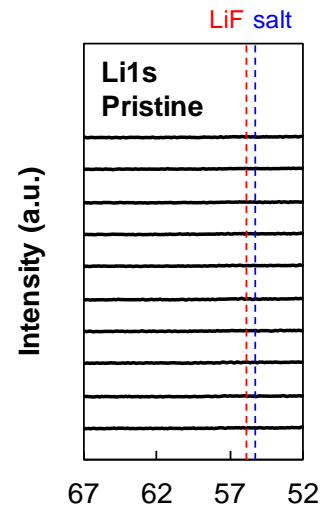

Binding energy (eV)
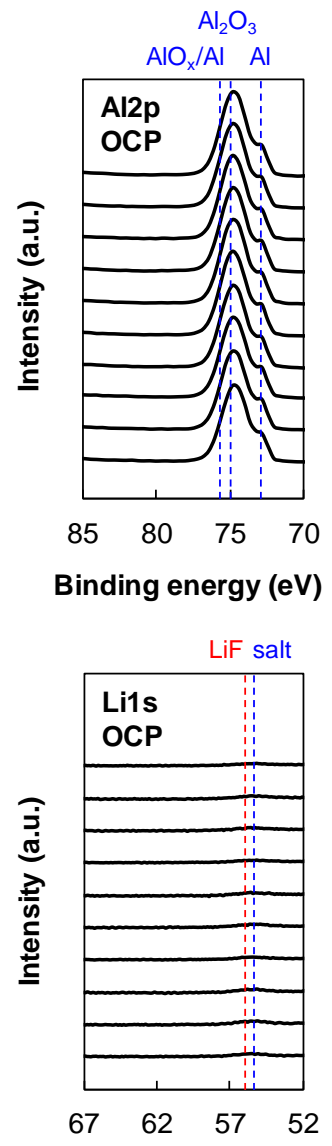

Binding energy (eV)
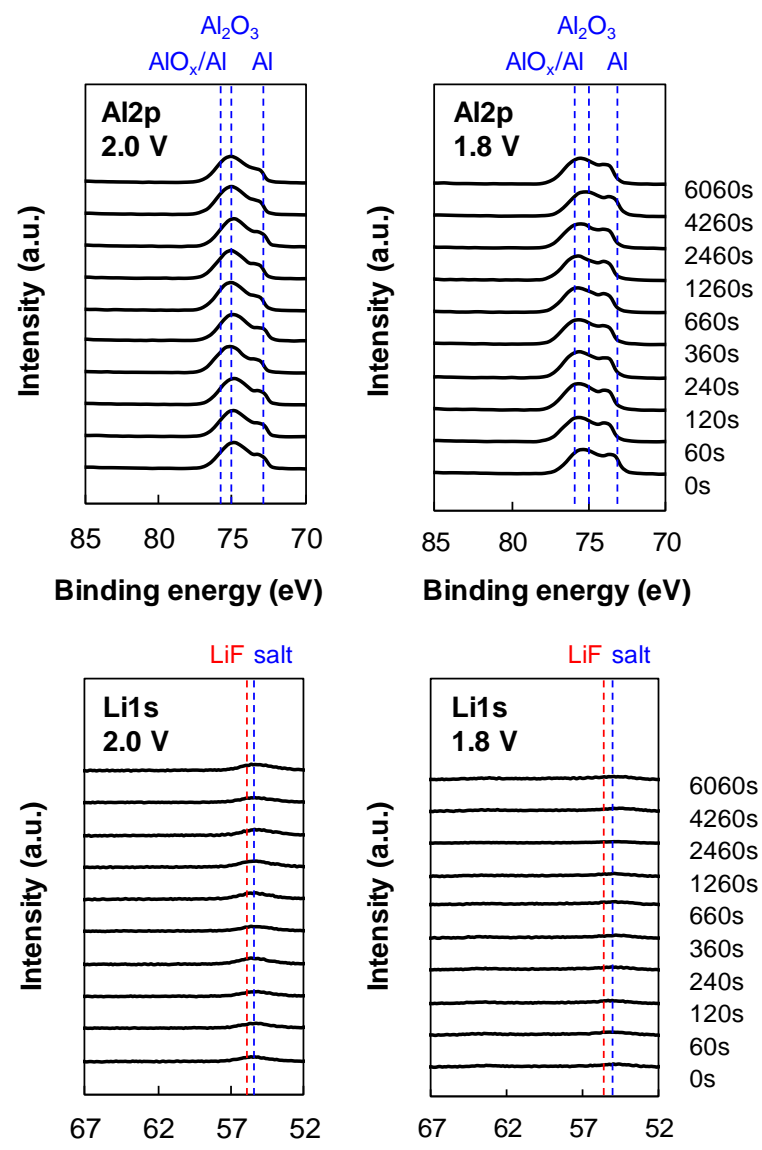

Binding energy (eV)

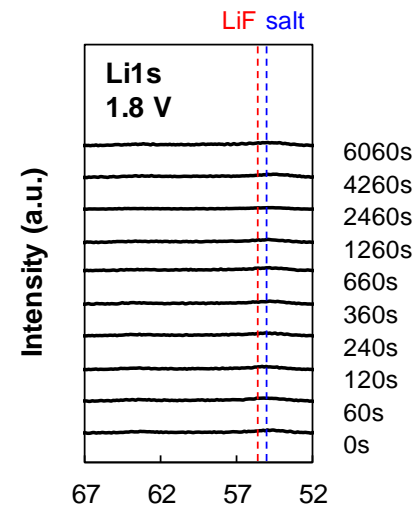

Binding energy (eV)

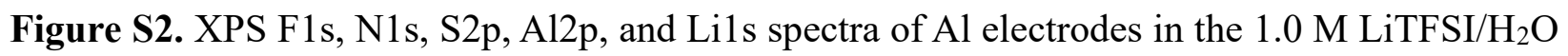
electrolyte. The $\mathrm{Al}$ electrodes were just immersed in the electrolyte for $24 \mathrm{~h}$ (denoted as OCP) or subjected to the potential sweep $\left(0.1 \mathrm{mV} \mathrm{s}^{-1}\right)$ down to $2.0 \mathrm{~V}$ or $1.8 \mathrm{~V} v s . \mathrm{Li}^{+} / \mathrm{Li}$. The $\mathrm{Al}$ electrodes were washed three times with DME before the XPS analysis. The depth profiles were obtained via $\mathrm{Ar}^{+}$sputtering at $1 \mathrm{kV}$ for $60 \sim 6060 \mathrm{~s}$. 


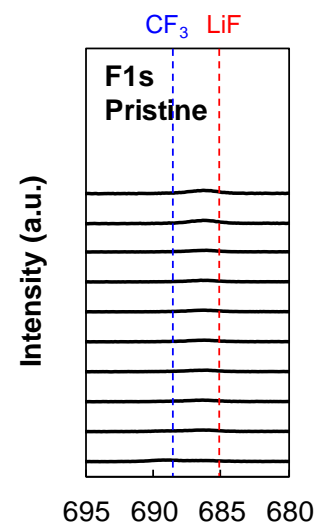

Binding energy (eV)

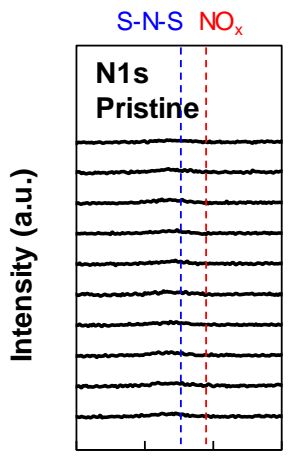

$407 \quad 402 \quad 397 \quad 392$

Binding energy (eV)

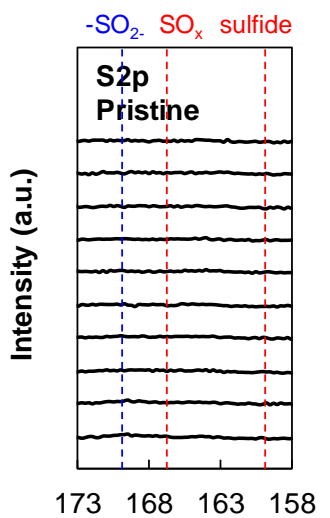

Binding energy (eV)

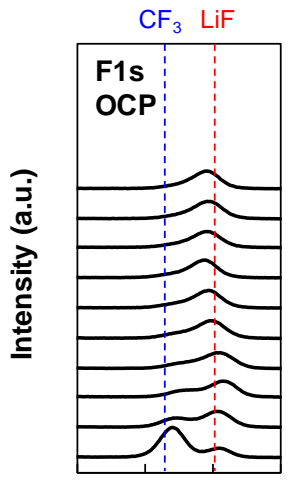

695690685680

Binding energy (eV)

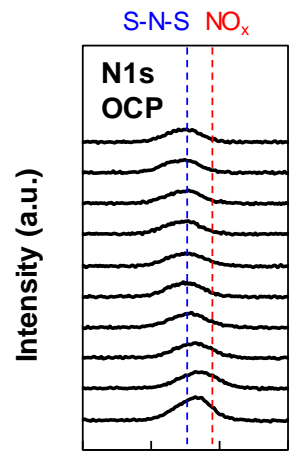

407402397392

Binding energy (eV)

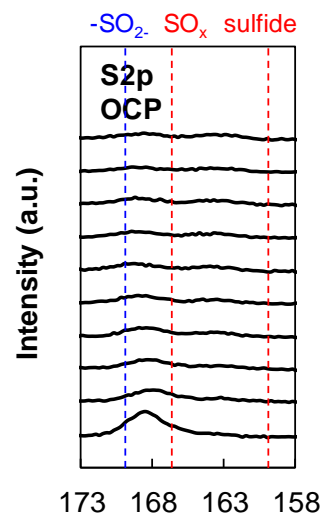

Binding energy (eV)

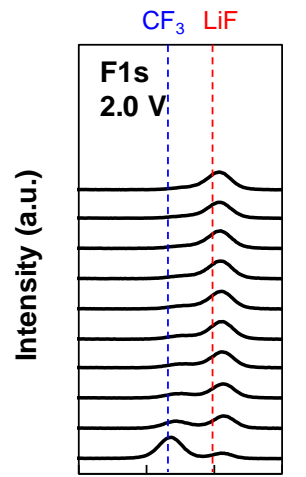

$695 \quad 690 \quad 685 \quad 680$

Binding energy (eV)

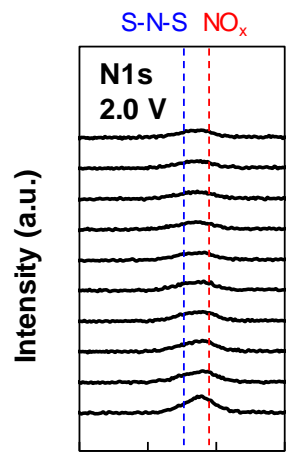

$407 \quad 402 \quad 397 \quad 392$

Binding energy (eV)
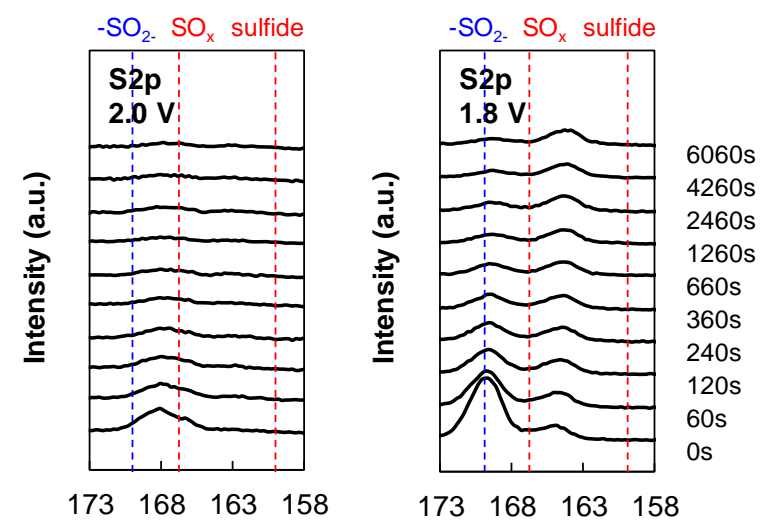

Binding energy $(\mathrm{eV})$

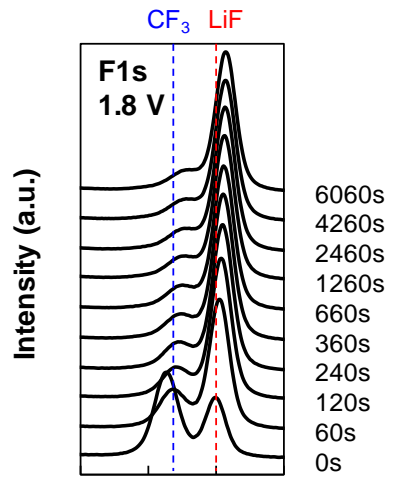

695690685680

Binding energy (eV)

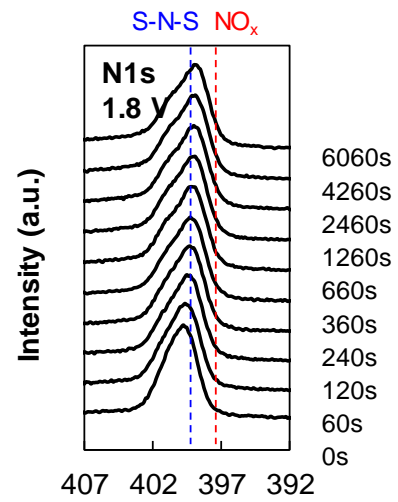

Binding energy (eV)

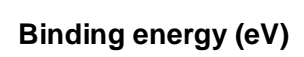



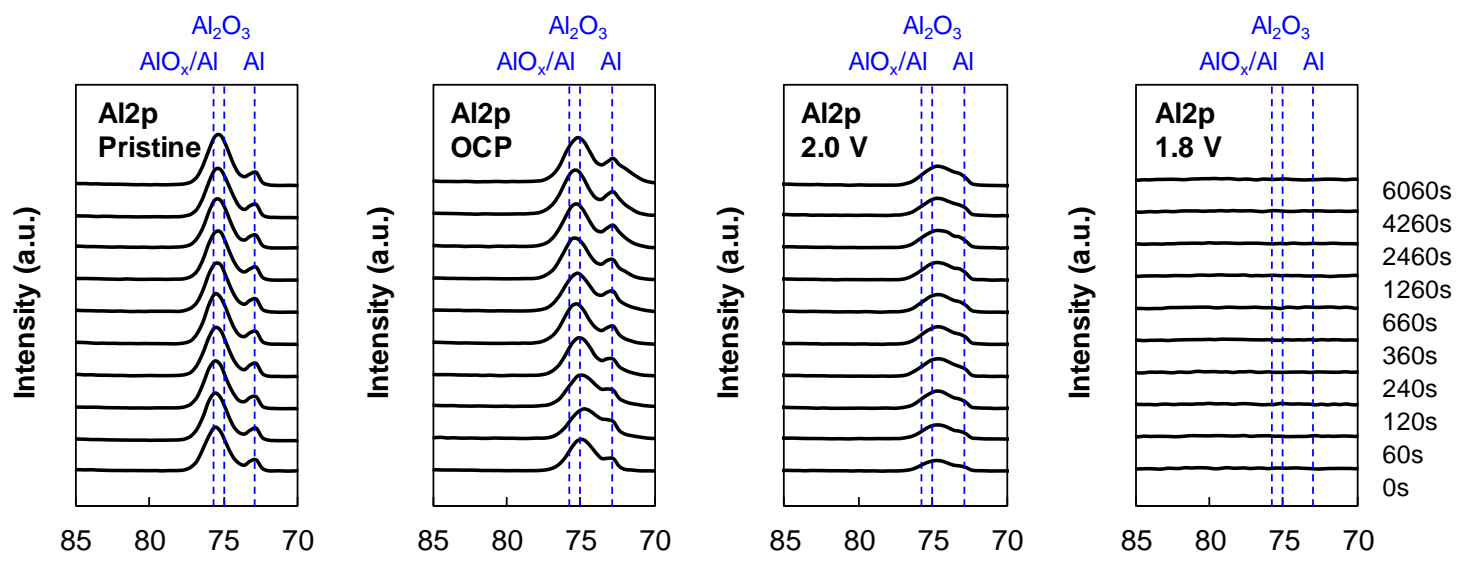

Binding energy (eV)

Binding energy (eV)

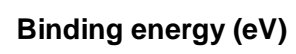

Binding energy (eV)
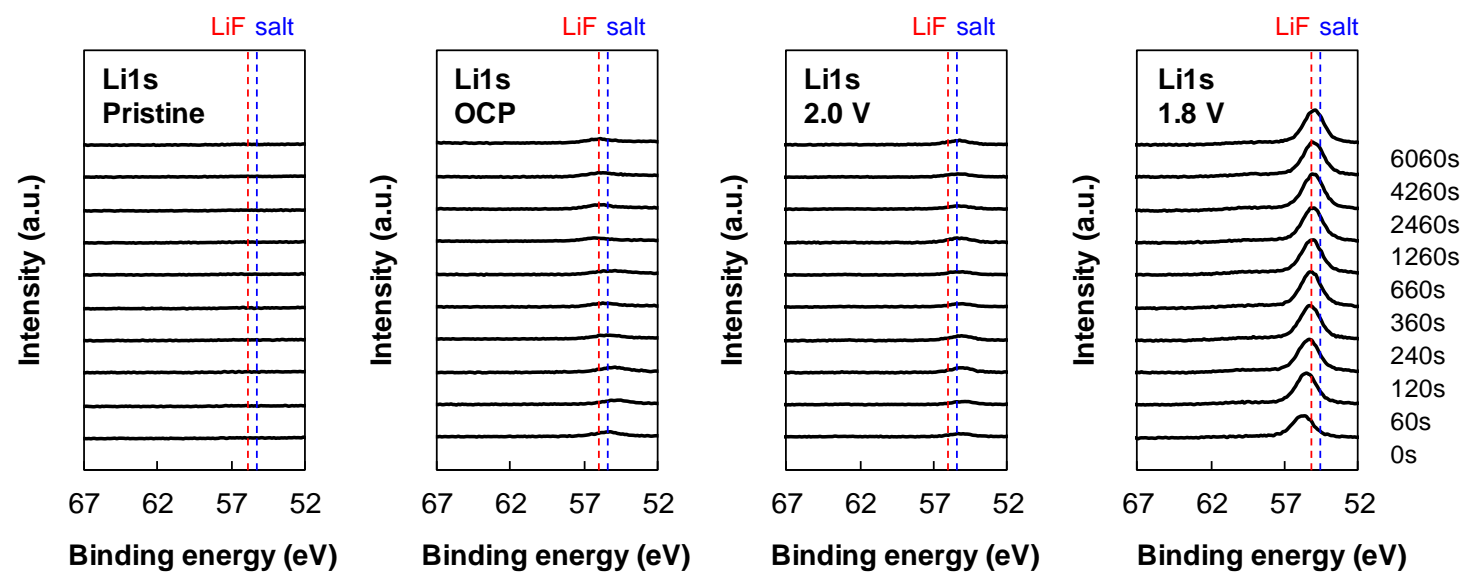

Figure S3. XPS F1s, N1s, S2p, A12p, and Li1s spectra of Al electrodes in the $\mathrm{Li}(\mathrm{TFSI})_{0.7}(\mathrm{BETI})_{0.3} \cdot 2 \mathrm{H}_{2} \mathrm{O}$ hydrate-melt electrolyte. The $\mathrm{Al}$ electrodes were just immersed in the electrolyte for $24 \mathrm{~h}$ (denoted as OCP) or subjected to the potential sweep $\left(0.1 \mathrm{mV} \mathrm{s}^{-1}\right)$ down to 2.0 $\mathrm{V}$ or $1.8 \mathrm{~V}$ vs. $\mathrm{Li}^{+} / \mathrm{Li}$. The $\mathrm{Al}$ electrodes were washed three times with DME before the XPS analysis. The depth profiles were obtained via $\mathrm{Ar}^{+}$sputtering at $1 \mathrm{kV}$ for $60 \sim 6060 \mathrm{~s}$. 


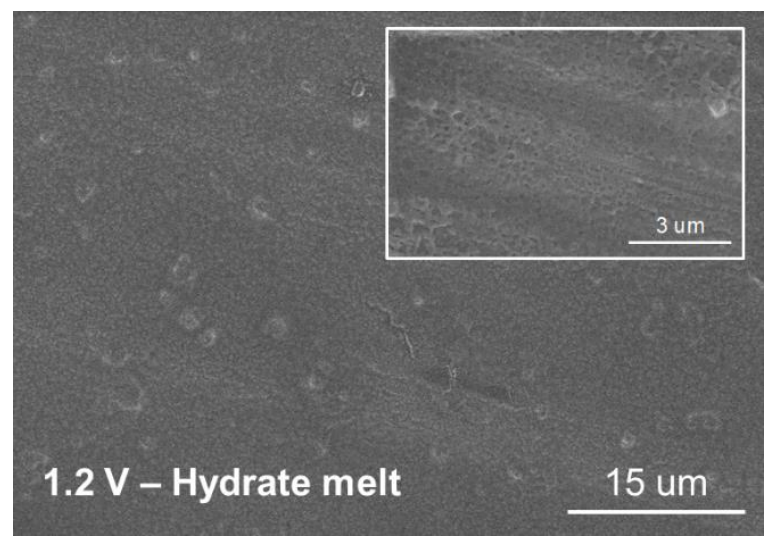

Figure S4. SEM images of the Al electrode after the potential sweep $\left(0.1 \mathrm{mV} \mathrm{s}^{-1}\right)$ down to $1.2 \mathrm{~V}$ vs. $\mathrm{Li}^{+} / \mathrm{Li}$ in the $\mathrm{Li}(\mathrm{TFSI})_{0.7}(\mathrm{BETI})_{0.3} \cdot 2 \mathrm{H}_{2} \mathrm{O}$ hydrate-melt electrolyte. The $\mathrm{Al}$ electrode was washed three times with DME before the SEM observation. 

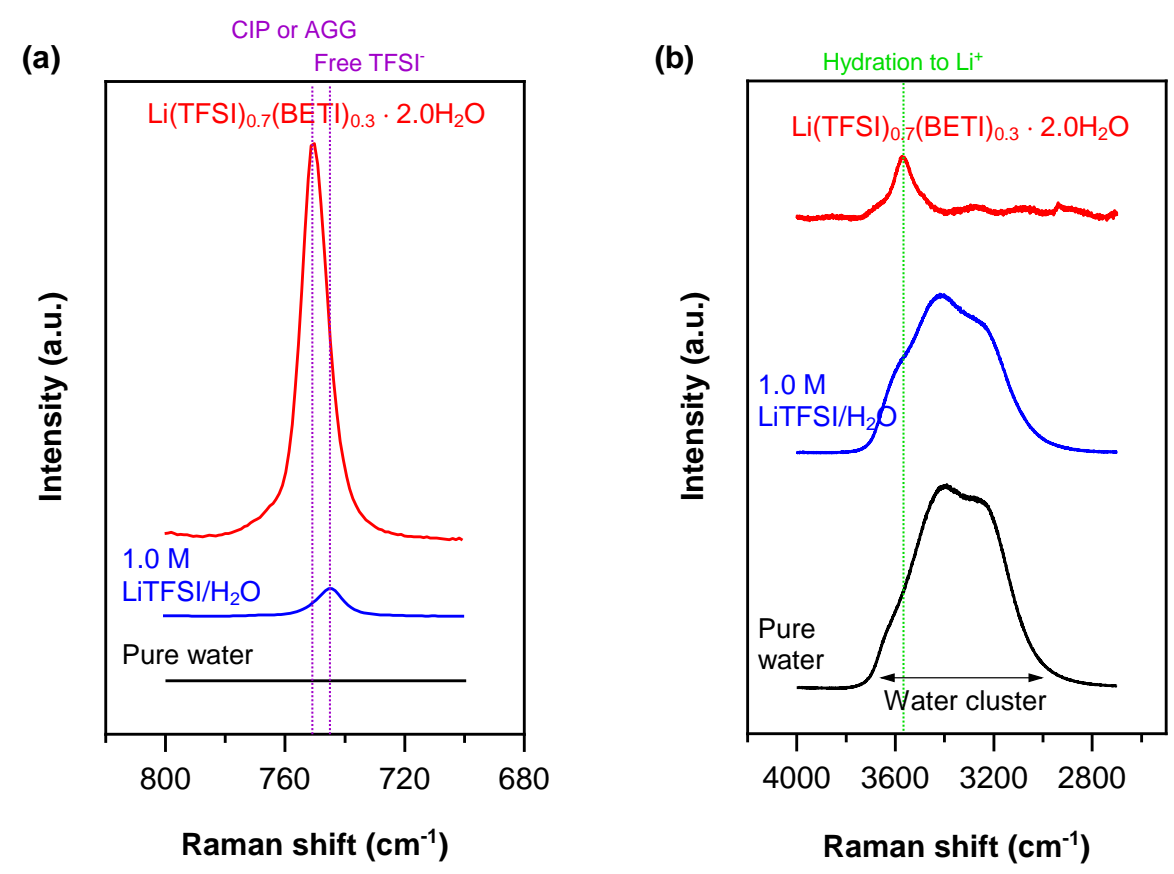

Figure S5. Raman spectra for (a) anion and (b) $\mathrm{O}-\mathrm{H}$ vibration of pure water, $1.0 \mathrm{M} \mathrm{LiTFSI} / \mathrm{H}_{2} \mathrm{O}$, and $\mathrm{Li}(\mathrm{TFSI})_{0.7}(\mathrm{BETI})_{0.3} \cdot 2 \mathrm{H}_{2} \mathrm{O}$ hydrate melt. The hydrate melt showed an extensive formation of ion pairs (or aggregates, AGG). The broad peak from water clusters disappeared in the hydrate melt, indicating the absence of free water molecules. 

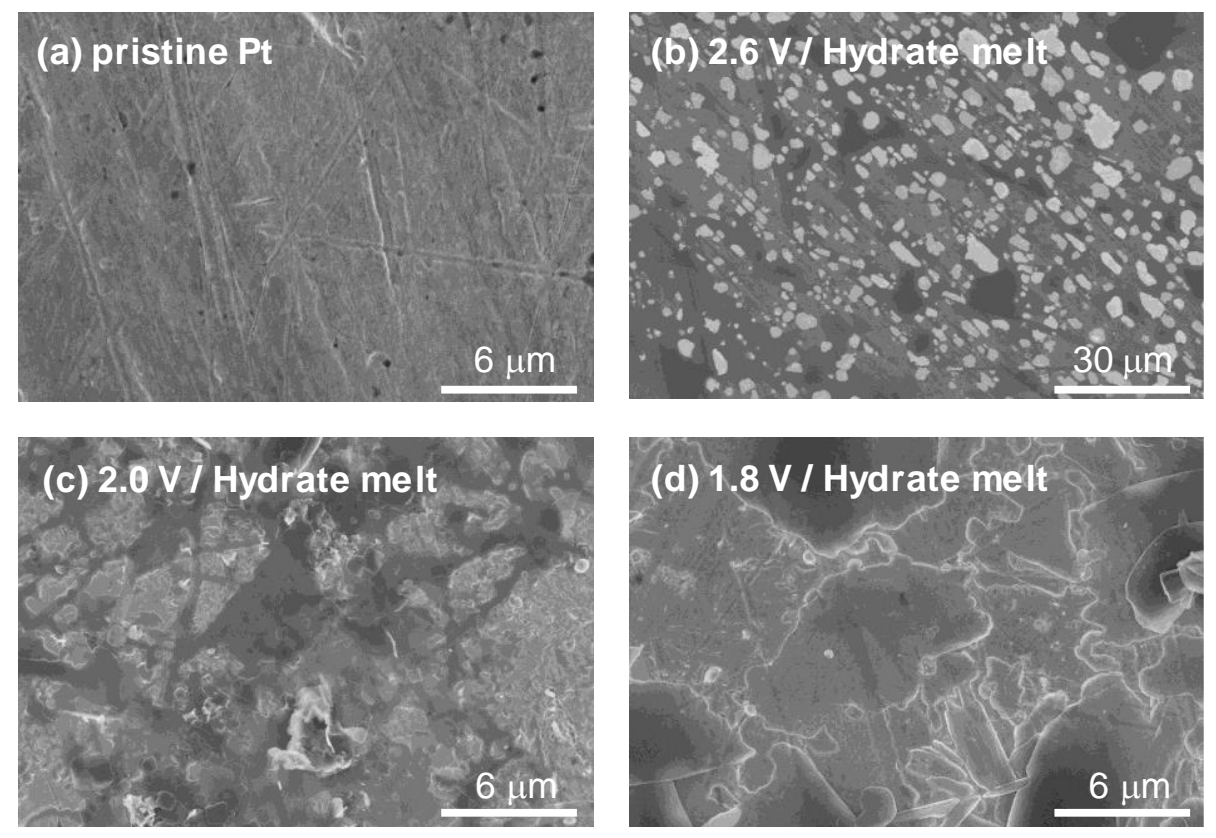

Figure S6. SEM images of the Pt electrodes after the potential sweep $\left(0.1 \mathrm{mV} \mathrm{s}^{-1}\right)$ down to 2.6, 2.0, and $1.8 \mathrm{~V}\left(v s . \mathrm{Li}^{+} / \mathrm{Li}\right)$ in the $\mathrm{Li}(\mathrm{TFSI})_{0.7}(\mathrm{BETI})_{0.3} \cdot 2 \mathrm{H}_{2} \mathrm{O}$ hydrate-melt electrolyte. The $\mathrm{Pt}$ electrode was washed three times with DME before the SEM observation. 